\title{
Optimization of titanium dioxide decorated by graphene quantum dot as a light scattering layer for enhanced dye-sensitized solar cell performance
}

\begin{abstract}
Titanium dioxide (TiO2) as a photoanode in dye-sensitized solar cells (DSSCs) has some drawbacks that reduce its photovoltaic performances i.e. low dye loading capacity and low light-harvesting efficiency. Therefore, $\mathrm{TiO} 2$ decorated by graphene quantum dot (GQD) as a light scatterer has been successfully fabricated via electrodeposition and drop-casting. The response surface methodology/central composite design was successfully utilized to optimize the preparation of photoanode with TiO2-GQD as a light scattering layer (LSL). A reduced quadratic model was successfully designed to predict the power conversion efficiency (PCE) accurately up to $97 \%$ with a $3 \%$ residual standard error. The TiO2-GQD LSL depicted a cluster of spherical nanoparticles on top of the photoanode that not only enhanced the light scattering effect but also improved the light-harvesting range from visible light to ultraviolet and nearinfrared range. The resultant $\mathrm{TiO} 2$ nanoparticles with TiO2-GQD LSL showed vast enhancement of PCE up to $66 \%$ from $3.06 \%$ to $5.01 \%$ due to a good synergistic effect.
\end{abstract}

